\title{
DESIGN AND ANALYSIS OF A LOW POWER BOOTSTRAPPED CMOS CIRCUIT
}

\author{
Neha Bharaj ${ }^{1}$ \\ ${ }^{1}$ Student, Electronics and Communication Engineering, Lovely Professional University, Punjab, India
}

\begin{abstract}
This paper describes bootstrapped circuit operated at low voltage. First I have described bootstrapped driver circuit that operated at ultra low supply voltage. Bootstrapping is a technique that improves the driving ability of digital circuit that operated on very low voltage. In this paper I have proposed bootstrapped driver circuit using CADENCE virtuoso at $180 \mathrm{~nm}$ as well as $90 \mathrm{~nm}$ and comparison of both circuit are mentioned and also layout of proposed bootstrapped driver circuit is also shown. The proposed bootstrapped driver circuit minimizes area overhead as compared to conventional bootstrapped driver circuit. Proposed driver circuit uses less number of transistors as compared to conventional driver circuit. Conventional bootstrapped driver circuit operated at $1 v$ to $1.5 \mathrm{v}$, if we try to reduce that supply voltage for low power application then actual working of driver circuit get disturbed at 180nm. The circuit which I have proposed at 180nm takes minimum supply voltage of $0.8 v$ and proposed driver circuit at 90nm take minimum supply voltage of $0.3 \mathrm{v}$. Final output voltage of bootstrapped driver circuit will be boosted output that is above VDD and below GND. Both proposed bootstrapped driver circuit give low power dissipation and less leakage current and also less delay as compared to conventional bootstrapped driver circuit. Proposed bootstrapped driver circuit offers $94.93 \%$ improvement in power dissipation.
\end{abstract}

Keywords: Ultra Low Voltage, CMOS Driver, Bootstrapped Drivers.

\section{INTRODUCTION}

Nowadays low power supply voltage is the trend of CMOS VLSI digital circuits [1] [2]. Since the threshold voltage of the CMOS devices cannot be scaled down accordingly with the power supply voltage. Minimum threshold voltage cannot scale down beyond defined limit for a standard cell library. Body biasing is another option but it needs extra supply voltage to body terminal. So, designing a CMOS circuit using a low power supply voltage for SOC applications has been challenging. In this paper design of low supply driver circuit has been explored. As we know the design of the driver circuit is the major step in determining the speed performance of a CMOS VLSI circuit with a large capacitive load. To raise and to lower the gate voltage of the output NMOS and PMOS device indirect bootstrap techniques is mainly used in the large load driver circuit using a low power supply voltage [3-5]. In indirect bootstrap technique, the bootstrap technique is applied at the gate of the output devices in a driver circuit. But when the bootstrap technique is applied at gate of the output device it is not effective in shortening the switching speed of the output. Since threshold voltage cannot be reduced with reduction in power supply in CMOS devices and the design of large load driver circuit is with reduced power supply is challenging.

The structure of this paper is as follows: Section 2 gives the scope of study, section 3 show conventional bootstrapped CMOS driver circuit, Section 4 shows design and implementation of proposed bootstrapped driver circuit, Section 5 shows the layout of proposed bootstrapped driver circuit, Section 6 shows post layout simulation results, section 7 will give results and discussion, section 8 gives conclusion of this whole study and at last references given.

\section{SCOPE OF THE STUDY}

The conventional bootstrapped driver circuit cannot work below supply voltage of $1 \mathrm{v}$. If we try to reduce that supply voltage in conventional bootstrapped driver circuit below $1 \mathrm{v}$, then actual working of driver circuit get disturbed. Moreover conventional bootstrapped driver uses more number of transistors that increases overall area of the circuit. The conventional bootstrapped driver uses bootstrap capacitor of large value that in turn increases area and power dissipation of the circuit.

To overcome the problem that occurs in conventional bootstrapped driver circuit I have modified that driver circuit. In conventional bootstrapped driver circuit value of bootstrapped capacitors are very large that is $\mathrm{Cp}=350 \mathrm{fF}$ and $\mathrm{Cn}=250 \mathrm{fF}$, whereas in proposed bootstrapped driver circuit value of bootstrapped capacitors are $\mathrm{Cp}=\mathrm{Cn}=17 \mathrm{fF}$. In conventional bootstrapped driver circuit when supply voltage $\mathrm{Vin}=1 \mathrm{v}$, then output positive level is boosted to Vout $=1.0001 \mathrm{v}$. Whereas in proposed bootstrapped driver circuit when supply voltage Vin $=1 \mathrm{v}$, then output positive level is boosted to Vout $=1.4 \mathrm{v}$. Moreover in conventional bootstrapped driver circuit the number of transistors used is more as compared to proposed bootstrapped driver circuit. So, less usage of transistors leads to reduction in area coverage. I have designed driver circuit using CADENCE virtuoso tool at $180 \mathrm{~nm}$ as well as $90 \mathrm{~nm}$ technology. The proposed bootstrapped driver circuit at $180 \mathrm{~nm}$ technology 
takes minimum supply voltage of $0.8 \mathrm{v}$ and boost output positive level to $1.17 \mathrm{v}$. The proposed bootstrapped driver circuit which I have designed at $90 \mathrm{~nm}$ technology takes minimum supply voltage of $0.3 \mathrm{v}$ and boosts the output positive level to $0.599 \mathrm{v}$ approximately double to VDD.

\section{CONVENTIONAL BOOTSRAPPED CMOS DRIVER}

Figure 1 below shows the circuit of conventional bootstrapped driver, which composed of fundamental segment and bootstrapped segment. In fundamental segment two PMOS devices are used that are PM0 and PM1 and two NMOS devices are used that are NM0 and NM1. In bootstrapped segment two PMOS device that are PM2 and PM3 and two NMOS devices are used that are NM2 and NM3. Transistors of inverter PM4 and NM4 and bootstrapped capacitors $\mathrm{C} 0$ and $\mathrm{C} 1$ are also included in bootstrapped segment. Here transistors NM2, NM3 and bootstrapped capacitor $\mathrm{C} 0$ are used for pull up transient operation, whereas transistor PM2, PM3 and bootstrap capacitor $\mathrm{C} 1$ are used for pull down transient operation.

In this circuit it is clear that bootstrap circuit is composed of two portions for pull-up and pull-down operations. Capacitor $\mathrm{Cp}$ and NMOS devices N1b/N2b are used for the bootstrapped pull-up operation and capacitor $\mathrm{Cn}$ and PMOS devices $\mathrm{P} 1 \mathrm{~b} / \mathrm{P} 2 \mathrm{~b}$ are used for the bootstrapped pull-down operation.

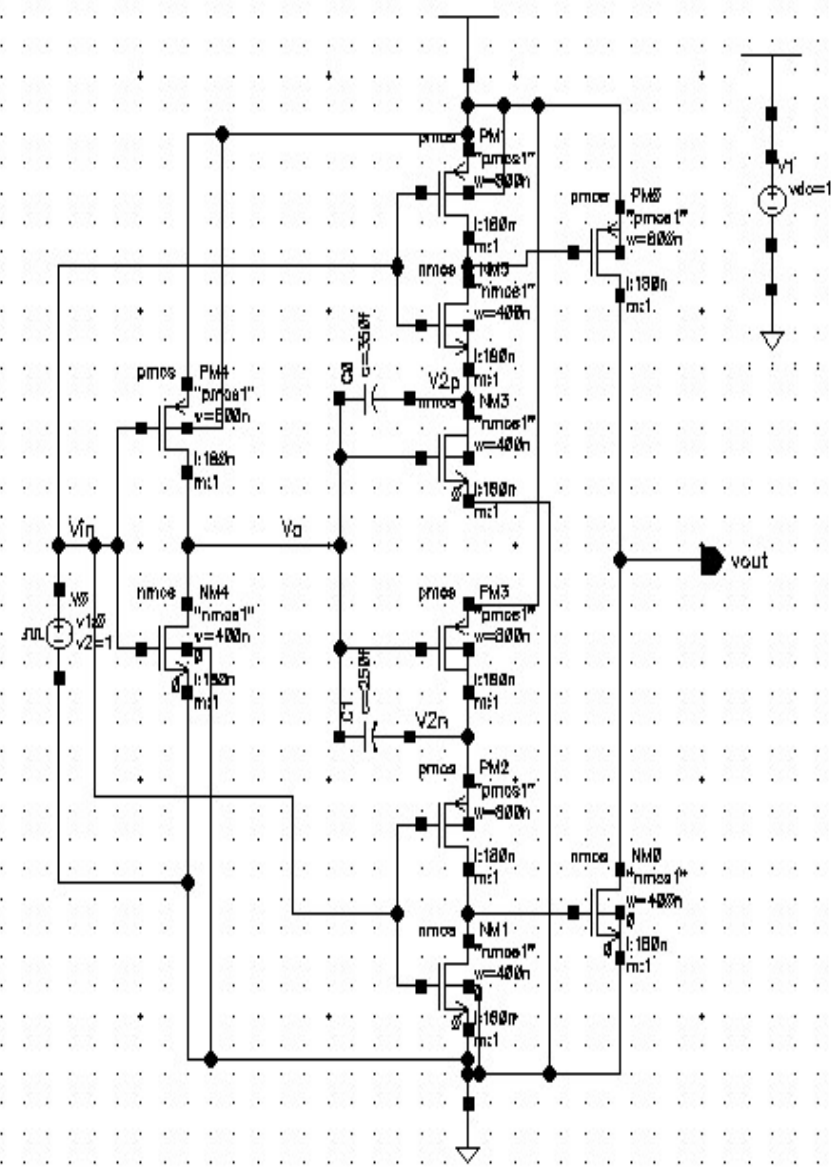

Figure 2 shows the transient waveforms of conventional bootstrapped driver circuit. As shown in figure 1 right side of bootstrapped capacitor $\mathrm{C} 0$ or node V $2 \mathrm{p}$ can go below $0 \mathrm{v}$ during the transient.

During pull up transient operation, the operation of this conventional bootstrapped driver circuit is divided into two periods regarding bootstrapped capacitor $\mathrm{C} 0$. First is charge build up period and second is bootstrapped period.

From figure 1 when input will get ON. The output of conventional bootstrapped driver Vout, which is indirectly driven by given to this circuit is Vin $=0 \mathrm{v}$, then output of inverter will be $1 \mathrm{v}$ which will make transistor NM2 and NM1 get OFF, transistor NM3 PM2 in the bootstrapped segment is $0 \mathrm{v}$.
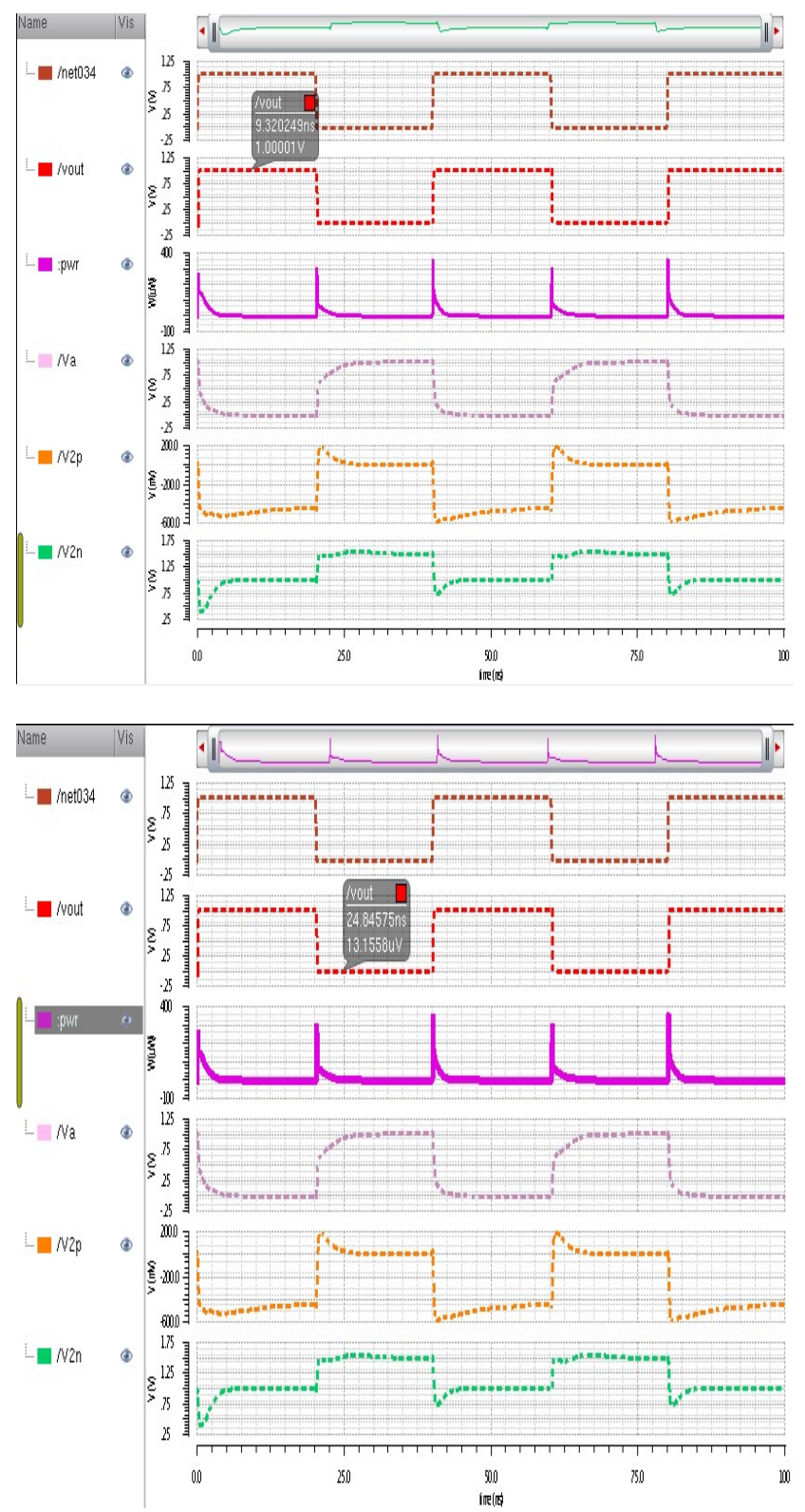

Fig-2: Waveform of conventional bootstrapped driver circuit

Fig-1: Conventional bootstrapped driver circuit 
On the other hand, transistor NM3 and bootstrapped capacitor $\mathrm{C} 0$ of bootstrapped segment are separated from the transistor PM1 and PM0 of fundamental segment. As a result the bootstrapped capacitor $\mathrm{C} 0$ has charge of $1 \mathrm{C} 0$ coulomb, since left side is pulled to $1 \mathrm{v}$ and right side is node $\mathrm{V} 2 \mathrm{p}$ to $0 \mathrm{v}$ because NM3 is $\mathrm{ON}$.

Now when supply voltage given Vin $=1 \mathrm{v}$, the output of inverter $\mathrm{Va}$ changes to $0.001 \mathrm{v}$. Due to which right side of bootstrapped capacitor $\mathrm{C} 0$ is disconnected from GND because transistor NM3 is OFF. Now this right side of bootstrapped capacitor $\mathrm{C} 0$ will get connected to gate of transistor PM0 because transistor NM2 is ON. Due the voltage change that occur at the left side of bootstrapped capacitor $\mathrm{C} 0$ from $1 \mathrm{v}$ to $0.001 \mathrm{v}, \mathrm{Va}$ changing from $1 \mathrm{v}$ to $0.001 \mathrm{v}$ then right side of bootstrapped capacitor $\mathrm{C} 0$ will change from $0 \mathrm{v}$ to $-0.506 \mathrm{v}$ i.e. node $\mathrm{V} 2 \mathrm{p}$ changes from $0 \mathrm{v}$ to $-0.506 \mathrm{v}$ means internal voltage undershoots. The extend of internal voltage undershoots is calculated by the ratio of bootstrapped capacitor $\mathrm{C} 0$ to the parasitic capacitance at the right side of $\mathrm{C} 0$. Due to this voltage undershoots the output voltage can switch at faster rate since gate voltage of transistor PM0 is driven at $-0.506 \mathrm{v}$. As shown in figure 2 output voltage can go up to full swing of $1.00001 \mathrm{v}$. The pull down transient operation has complementary working as pull up transient. In this conventional bootstrapped driver circuit value of bootstrapped capacitors are $\mathrm{C} 0=350 \mathrm{fF}, \mathrm{C} 1$ $=250 \mathrm{fF}$. Sizing of all PMOS transistors width/length = $800 \mathrm{~nm} / 180 \mathrm{~nm}$, all NMOS transistors width/length = $400 \mathrm{~nm} / 180 \mathrm{~nm}$.

\section{PROPOSED BOOTSTRAPPED DRIVER AT}

\section{NM TECHNOLOGY}

This modified bootstrapped driver circuit when used at $180 \mathrm{~nm}$ technology of cadence virtuoso the minimum input supply voltage which we can give to this circuit is $0.8 \mathrm{v}$ or $800 \mathrm{mv}$. In this modified bootstrapped driver circuit input supplied is pulse with period of $20 \mathrm{~ns}$ and pulse width of 10ns. Here two bootstrapped capacitors are used i.e. $\mathrm{Cp}=\mathrm{Cn}=17$ femto farad. Using this output voltage boost to 1.2 volt. In the circuit diagram of modified bootstrapped driver $\mathrm{Cp}$ and $\mathrm{Cn}$ are bootstrapped capacitor PM1 and NM2 are transistor for $\mathrm{Cp}$ precharge and $\mathrm{Cn}$ predischarge. V2p and V2n are the boosted nodes. Output from transistors PM3 and NM3 will act as inverter output and this output $\mathrm{Va}$ is used to control PM2 and NM1. Final vout is the output voltage of modified bootstrapped driver that is boosted above Vdd and below GND. Using this driver output we can drive further circuitry that work on low voltages. When Vin is transiting from high to low, initially node V2n is at $0 \mathrm{v}$. After transiting from high to low i.e. when $\mathrm{Vin}=0 \mathrm{v}$, then node V2n will be boosted to $-587.718 \mathrm{mv}$. At this moment transistor PM2 turned OFF and NM1 turned ON. Therefore boosted signal at node $\mathrm{V} 2 \mathrm{n}$ pass from NM1 and reach at Vout.

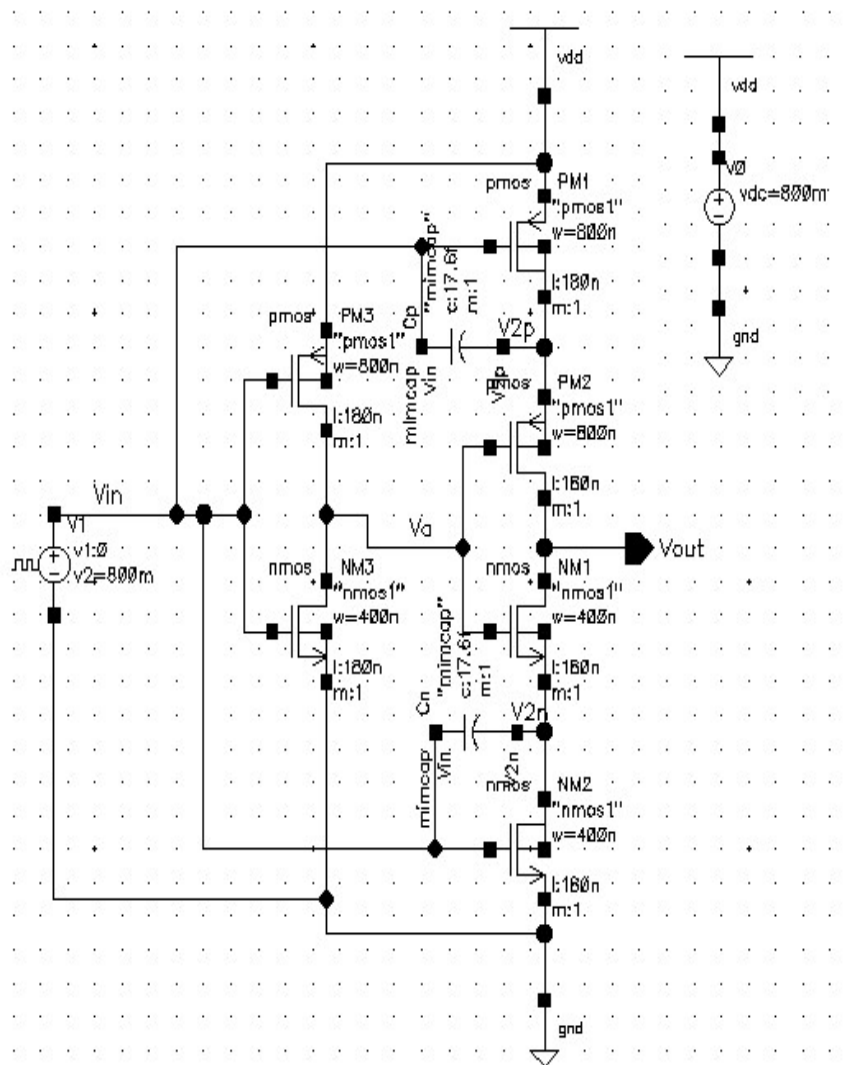

Fig-3: Proposed bootstrapped driver circuit at 180nm

At the same time when Vin $=0 \mathrm{v}$, the transistor PM1 is $\mathrm{ON}$ to precharge node V2p to VDD is $0.8 \mathrm{v}$ or $800 \mathrm{mv}$. However NM2 is turned ON reversely causing the reverse current flow to charge V2n, when Vin is low or $0 \mathrm{v}$. At the end of period node V2n still holds the charge $-250.193 \mathrm{mv}$. Now when Vin is HIGH, transistor PM1 gets OFF, Node V2p is boosted above VDD i.e. upto $1.4 \mathrm{v}$. At the end of period it still holds charge $1.197 \mathrm{v}$. At the same time as vin is $0.8 \mathrm{v}$, then PM2 turned ON and NM1 turned OFF. So node V2p is boosted upto $1.475 \mathrm{v}$ and this will go to Vout through transistor PM2 At the same moment NM2 is turned ON it will precharge capacitor $\mathrm{Cn}$ to $-12.9994 \mathrm{uv}$ or below GND. So final output voltage which we get after complete swing of Vin, when it transit from LOW to HIGH and HIGH to LOW is when Vin is $0.8 \mathrm{v}$ then output voltage Vout will boost above $\mathrm{Vdd}$ to $1.3 \mathrm{v}$. When Vin is $0 \mathrm{v}$ then output voltage will boost below Gnd to $-366.843 v$. Figure 4 shows the simulated waveform of proposed bootstrapped driver circuit at $180 \mathrm{~nm}$ technology.

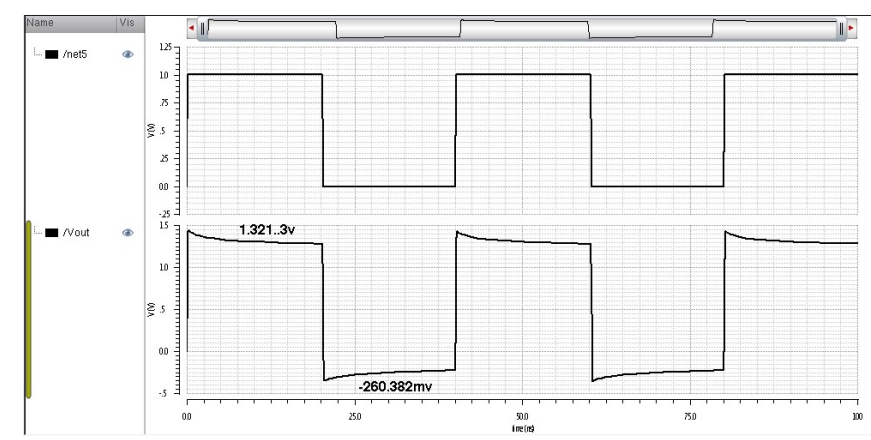

Fig-4: Output waveform of proposed bootstrapped driver 


\section{LAYOUT OF PROPOSED BOOTSTRAPPED DRIVER}

I have made layout of modified bootstrapped driver at CADENCE Virtuoso 180nm technology using bootstrapped capacitors of 17 femto farad. These two bootstrapped capacitors are mimcap having width of 4um and length of 4um. This is the minimum size capacitor which I have used in my modified design. If we increase these capacitor value then our capacitor sizes also get increased which make layout are to get increased. According to this modified bootstrapped driver layout, layout area covered is

Layout area $=13.345 \mathrm{um} * 12.745 \mathrm{um}$

$=170.082025 * 10^{-12} \mathrm{~m}^{2}$ or $0.170 \mathrm{~nm}^{2}$

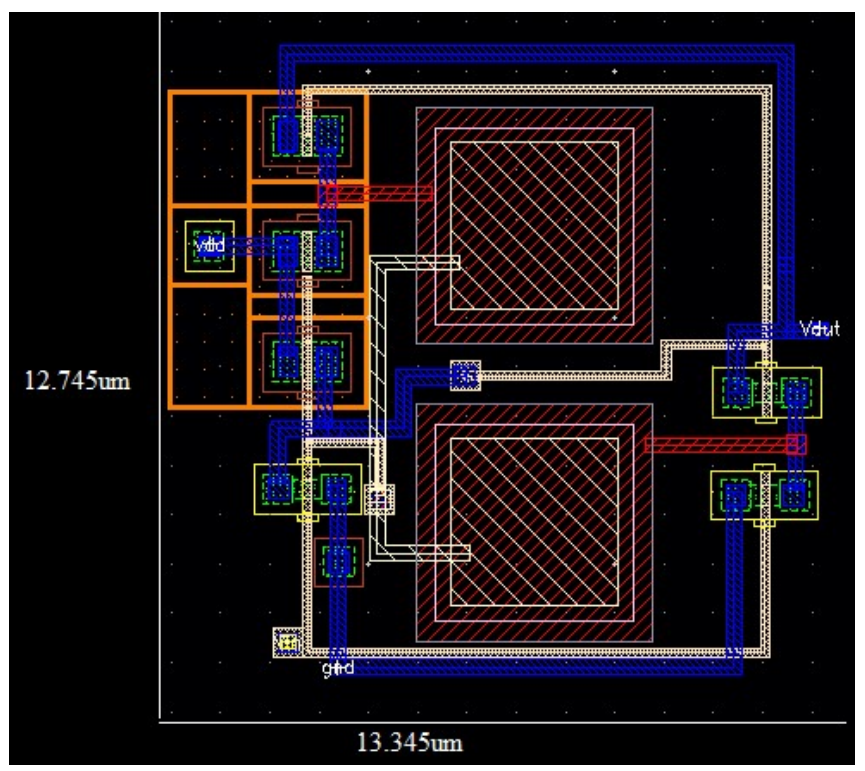

Fig-5: Layout of proposed bootstrapped driver circuit

\section{POST LAYOUT SIMULATION OF PROPOSED BOOTSTRAPPED \\ DRIVER \\ CIRCUIT}

Figure 6 below shows the configuration circuit of proposed bootstrapped driver circuit in which Vin $=1 \mathrm{v}$ is supplied.
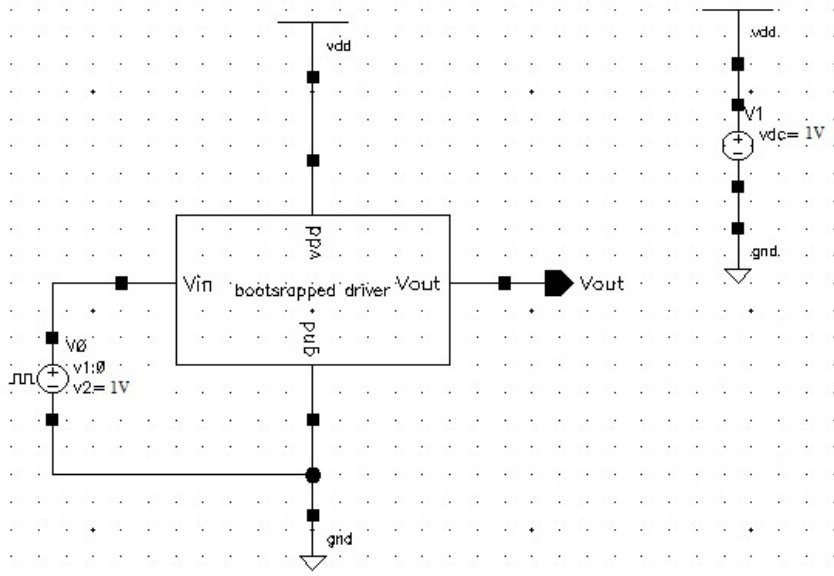

Fig-6: Configuration circuit of proposed bootstrapped driver circuit
Figure 7 below shows the post layout simulation response of proposed bootstrapped driver circuit at 180nm technology.

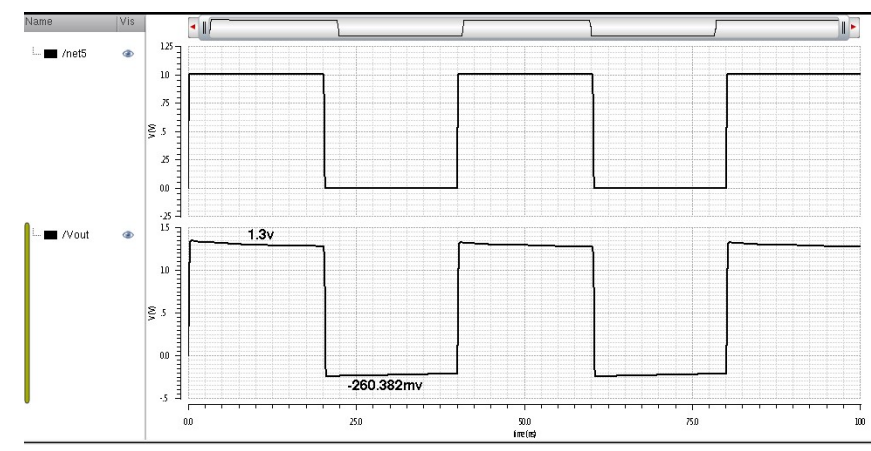

Fig-7: Output response of post layout simulation of proposed bootstrapped driver

\section{RESULTS AND DISCUSSION}

In figure 8 below shows the comparison of output voltages in bootstrapped driver circuits designed at CADENCE virtuoso $180 \mathrm{~nm}$ and $90 \mathrm{~nm}$ technologies. From this figure it is clear that proposed bootstrapped driver circuit gives better results as compared to conventional bootstrapped driver circuit.

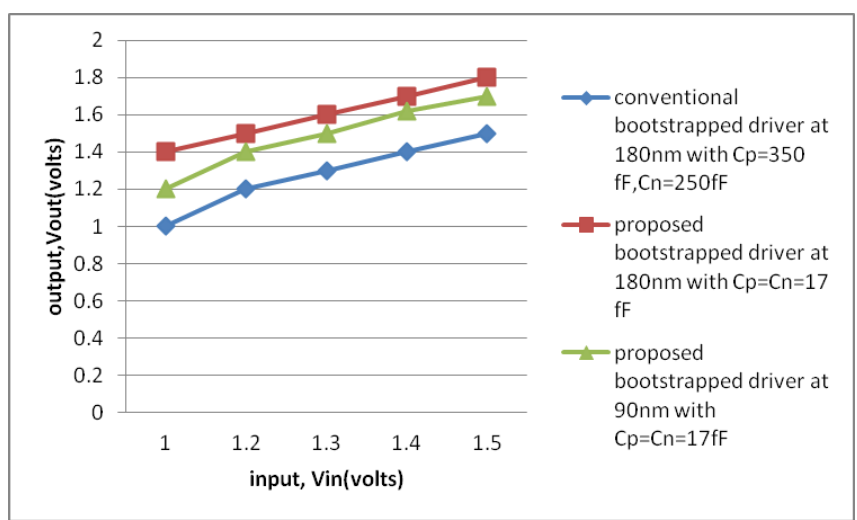

Fig-8: Comparison of vin and vout of bootstrapped driver circuits

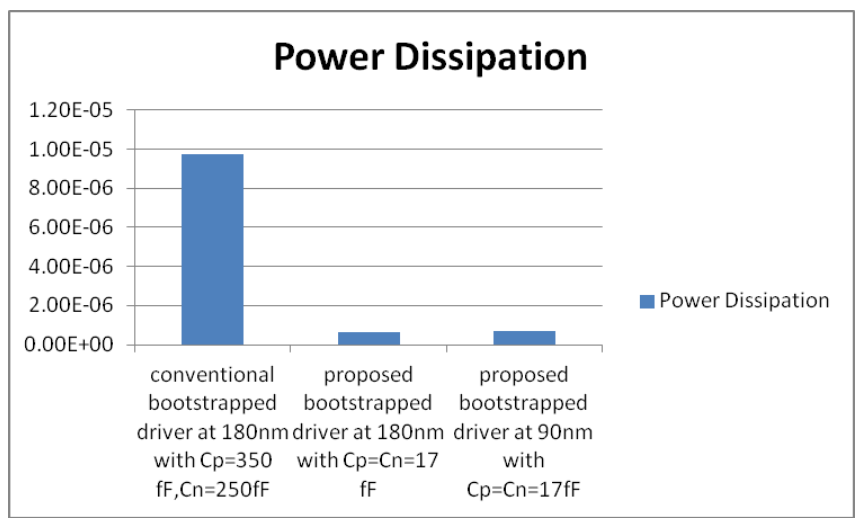

Fig-9: Comparison of power dissipation of bootstrapped driver circuits

Figure 9 shows the comparison of power dissipation occurs in different bootstrapped driver circuits. In conventional bootstrapped driver circuit power dissipation is $9.73 \mathrm{E}-06$, in 
proposed bootstrapped driver at $180 \mathrm{~nm}$ power dissipation calculated is $6.57 \mathrm{E}-07$ and in proposed bootstrapped driver at $90 \mathrm{~nm} \mathrm{PD}=6.83 \mathrm{E}-07$

Figure 10 shows the comparison of delay occurs in different bootstrapped driver circuits. In conventional bootstrapped driver circuit delay $=20.08 \mathrm{E}-9$, in proposed bootstrapped driver circuit at $180 \mathrm{~nm}$ delay $=20.07 \mathrm{E}-9$ and in proposed bootstrapped driver circuit at $90 \mathrm{~nm}$ delay $=20.03 \mathrm{E}-9$.

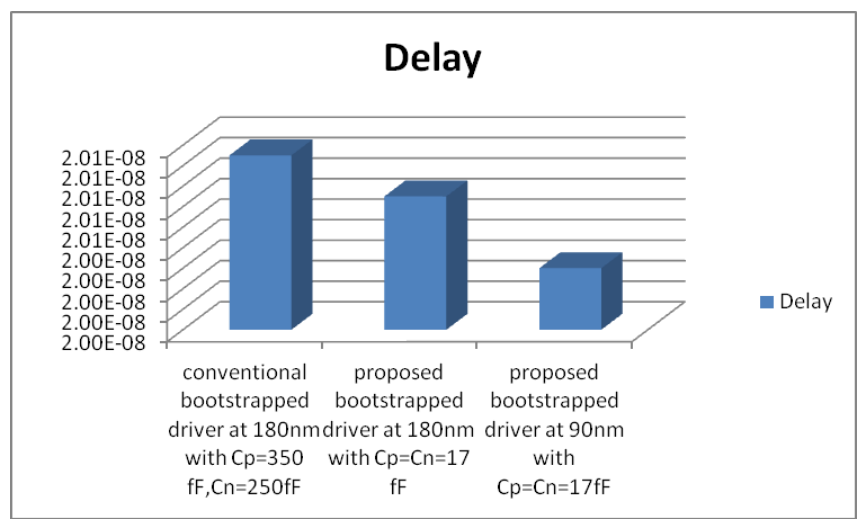

Fig-10: Comparison of delay occur in bootstrapped driver circuits
Figure 11 shown below compares the leakage current occurs in different bootstrapped driver circuits. In conventional bootstrapped driver circuit leakage current $=3.50 \mathrm{E}-12$, in proposed bootstrapped driver circuit at $180 \mathrm{~nm}$ leakage current $=3.49 \mathrm{E}-12$ and in proposed bootstrapped driver circuit at $90 \mathrm{~nm}$ leakage current $=9.78 \mathrm{E}-11$.

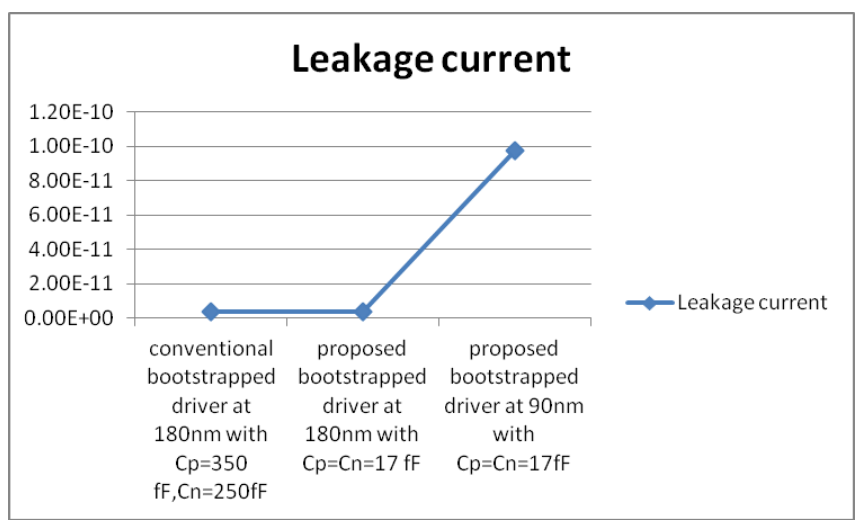

Fig-11: Comparison of leakage current occur in bootstrapped driver circuits

Table-1: Transistors sizing in bootstrapped driver

\begin{tabular}{|l|l|l|l|}
\hline PARAMETER & $\begin{array}{l}\text { PMOS } \\
\text { W/L }\end{array}$ & $\begin{array}{l}\text { NMOS } \\
\text { W/L }\end{array}$ & $\begin{array}{l}\text { Total number of } \\
\text { transistor } \\
\text { PMOS/NMOS }\end{array}$ \\
\hline $\begin{array}{l}\text { Conventional bootstrapped driver at } 180 \mathrm{~nm} \text { with } \mathrm{Cp}=350 \\
\mathrm{fF}, \mathrm{Cn}=250 \mathrm{fF}\end{array}$ & $800 \mathrm{~nm} / 180 \mathrm{~nm}$ & $400 \mathrm{~nm} / 180 \mathrm{~nm}$ & $5 / 5$ \\
\hline Proposed bootstrapped driver at $180 \mathrm{~nm}$ with $\mathrm{Cp}=\mathrm{Cn}=17 \mathrm{fF}$ & $800 \mathrm{~nm} / 180 \mathrm{~nm}$ & $400 \mathrm{~nm} / 180 \mathrm{~nm}$ & $3 / 3$ \\
\hline Proposed bootstrapped driver at $90 \mathrm{~nm}$ with $\mathrm{Cp}=\mathrm{Cn}=17 \mathrm{fF}$ & $360 \mathrm{~nm} / 150 \mathrm{~nm}$ & $120 \mathrm{~nm} / 150 \mathrm{~nm}$ & $3 / 3$ \\
\hline
\end{tabular}

\section{CONCLUSION}

In this paper I have explained about bootstrapped technique and shown how we can use that technique in driver circuits. The proposed driver circuit gives better results compared to conventional driver circuit with low power dissipation less delay and less leakage current. Proposed bootstrapped driver circuits are designed at CADENCE virtuoso $180 \mathrm{~nm}$ as well as $90 \mathrm{~nm}$ technology. From layout of proposed bootstrapped driver circuit it is verify that proposed driver circuit occupy less area. In GDI technology which cascaded transistor (PTL) there is a serious problem of degradation of output level after some stage, level restoration logic is required to achieve the output voltage level. Above bootstrap circuit can also be used for logic restoration devices to maintain the constant voltage for logic.

\section{REFERENCES}

[1]. J. B. Kuo et al, "Low-Voltage SOI CMOS VLSI Devices and Circuits," John Wiley Intersicence, New York, ISBN 0471-417777, 2001.

[2]. J. B. Kuo et al, "Low-Voltage CMOS VLSI Circuits," John Wiley Interscience, New York, ISBN 0471-321052, 1999.
[3]. LOU, J.H., and KUO, J.B.: 'A 1.5-V fullswing bootstrapped CMOS large capacitive-load driver 'circuit suitable for low-voltage CMOS VLSI', IEEE. JT Solid-state Circuits, 1997, 32, pp. 119-121

[4]. LAW, C.F., YEO, K.S., and SAMIR, R.S.: 'Sub-I V bootstrapped CMOS driver for giga-scale-integration era', Electro. Lett., 1999, 35, pp. 392-393

[5]. P. C. Chen and J. B. Kuo, "Sub-1V CMOS Driver Circuit using Direct Bootstrap Technique," Electronics Letters, pp. 265-266, Vol. 38, No. 6, March 2002.

[6]. YEO, K.S., MA, J.G., and DO, M.A.: 'Ultra-lowvoltage bootstrapped CMOS driver for high performance applications', Electro. Lett., 2000, 36, pp. 706-707

[7]. J. C. Garcia, J. A. Montiel-Nelson, and S. Nooshabadi, "A single-capacitor bootstrapped power-efficient CMOS driver," IEEE Trans. Circuits Syst. II, Exp. Briefs, vol. 53, no. 9, pp. 877-881, Sep. 2006.

[8]. J. W. Kim and B. S. Kong, "Low-voltage bootstrapped CMOS drivers with efficient conditional bootstrapping," IEEE Trans. Circuits Syst. II, Exp. Briefs, vol. 55, no. 6, pp. 556-560, Jun. 2008.

[9]. J. C. Garcia, J. A. Montiel Nelson, J. Sosa and H. Navarro, A direct bootstrapped CMOS large capacitive load 
driver circuit, Design, Automation and Test in Europe Conference and Exhibition, vol. 1, pp. 680.681, Feb. 2004.

[10]. B. S. Kong and Y. H. Jun, "Power-efficient lowvoltage bootstrapped CMOS latched driver," Electron. Letter, vol. 35, no. 24, pp. 2113-2115.

[11]. P. Favrat, P. Deval, and M. J. Declercq, "A highefficiency CMOS voltage doubler", IEEE Custom Integrated Circuits Conf., 1997, pp. 259-262, 1997

[12]. J. F. Dickson, "On chip high voltage generation in NMOS integrated circuits using an improved voltage multiplier technique", IEEE J. Solid State Circuits, vol. SC11, no 3, June 1976, pp. 374-378.

[13]. J. H. Lou and J. B. Kuo, '1.5V CMOS and BiCMOS Bootstrapped Dynamic Logic Circuits," Proc. IEEE International Symp. VLSI Tech. Systems, Applications, pp. 279 282, Taipei, 1997.

[14]. B. H. Calhoun, A. Wang, and A. P. Chandrakasan, "Modeling and sizing for minimum energy operation in subthreshold circuits," IEEE J. Solid-State Circuits, vol. 40, no. 9, pp. 1178-1186, Jan. 2005.

\section{BIOGRAPHIE}

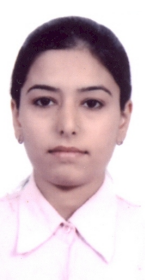

Neha Bharaj (F'28) did B.tech in electronics and communication from lovely institute of technology in 2011 and M.tech in electronics and communication from lovely professional university in 2014 . Her research focuses on operating bootstrapped CMOS circuits at low voltage. 